\title{
Conical Diffraction Based Super-resolution System for Fluorescence Microscopy: System Description and Demonstration visualizing Biological Objects
}

\author{
Gabriel Y. Sirat ${ }^{1}$, Spencer Shorte ${ }^{2}$, L.Philippe (Ori) Braitbart ${ }^{1}$, Lionel Moisan ${ }^{3}$, Jean Yves Tinevez ${ }^{2}$, \\ Julien Caron ${ }^{1}$ and Clément Fallet ${ }^{1}$ \\ 1. Bioaxial SAS, Paris Biotech Santé, 24 rue du Faubourg Saint Jacques, 75014 Paris, FRANCE \\ 2. PFID, Imagopole, Institut Pasteur, 25-28, rue du Docteur Roux 75015 Paris, FRANCE \\ 3. Université Paris Descartes, Laboratoire MAP5, 45 rue des Saints Pères, 75006 Paris, FRANCE
}

We present a novel technique for super resolution imaging in live-cell microscopy, based on conical diffraction in biaxial crystals. The industrial prototype that implements this technique is used routinely since August 2012 in the imaging facilities of the Institut Pasteur, Paris, to image with super-resolution, biological processes.

Conical refraction [1] or diffraction [2] describes the propagation of light along an optic axis of a biaxial crystal. The conical diffraction based super resolution system for fluorescence microscopy consists of a controllable polarizer, a thin biaxial crystal and a controllable analyzer [3]. By electronically switching sequentially the polarizer and analyzer, a light beam passing through the optical module creates a sequence of singular compact illumination patterns across the sample (Figure 1). The patterns illuminate a tiny volume, the illuminated region, the size of which is delimited as in routine confocal microscopy. The industrial prototype is mounted, as an add-on to a standard confocal microscope, in place of the illumination fiber. The system includes a bypass to acquire also directly confocal images.

In the super resolution mode, the sequence of compact illuminations is scanned on the fluorescent sample, and the emitted light from the sample recorded on an EMCCD camera yielding a stack of microimages. Each micro-image contains energy contributions from emitting objects, differing for each unique illumination pattern. Dependent upon its position each emitting object contributes energy differently for each illumination pattern resulting in a distinct series of micro-images each a function of the illuminated region and the coordinate position of each object. From the image-series the distribution of fluorescent objects is reconstructed using a model-based technique that matches the data to a simulated response calculated from the illumination Point Spread Function, the geometrical parameters of the system and the predicted object distributions. The super resolution information arises from the combination of the super resolution localized features created by the sequence of singular illuminations, using applied positivity and sparsity constraints [4], and the object-model, which involves a limited number of variables relative to the strong redundancy of the data. Additionally, for relatively sparse scenes, with few objects per illuminated region (up to 3-5), multi-localization algorithms can be used, that create composite images with only one object per image, allowing the use of high precision localization algorithms on each one of the objects.

Using this approach several different samples have been targeted for validation studies, including: synthetic beads, and biological substrates such as actin filaments, microtubules, and FISH samples. These validation studies have helped establish calibration and systematic operational procedures that are now integrated to the method. Consequently, from January 2013, a comprehensive comparative analysis has been proposed and implemented, to assess the system performance relative to other imaging modalities. The results measured by the system can be compared, in a reasonable time frame, to 
widefield imaging, to confocal imaging, to SIM (structured illumination microscopy), and eventually to SEM (scanning electron microscopy), on the exact same sample region of interest. Preliminary results of the comparative study will be presented. A measurement of the light dose and of the power level of the system has been performed and is to be completed by a study of the photodamage and photobleaching characteristics of the system.

Additional improvements have been identified and will be implemented in the beta version, scheduled in the first half of 2013. The beta systems will be designed for operation by biological scientists, after a short training period, using a procedure, marginally different from that of a conventional confocal microscope.

The system is characterized by its high light efficiency; each photon emitted by the sample, in the objective acceptance cone, is detected with high quantum efficiency by the EMCCD. This feature has been preliminarily confirmed experimentally by a low photobleaching of the samples measured, including samples known to be sensitive to photobleaching. The operation of the system is very simple, does not require special procedure for the preparation of the sample, is compatible with any fluorophore, and permits super resolution imaging on living, fragile specimens. All these features suggest imaging based upon conical diffraction is a promising tool for super resolution studies on live objects, and has potential as a powerful utility for super-resolution time-lapse imaging.

[1] WR Hamilton. Trans. Royal Irish. Acad. 17: 1-14 (1837).

[2] Berry, M.; M. Jeffrey. Conical diffraction: Progress in Optics 50: 13-50 (2007).

[3] Sirat, G.Y. Patent application PCT/FR2011/000555.

[4] E. J. Candès, J. Romberg and T. Tao, Stable signal recovery from incomplete and inaccurate measurements. Comm. Pure Appl. Math. 59, pp. 1207-1223, 2006

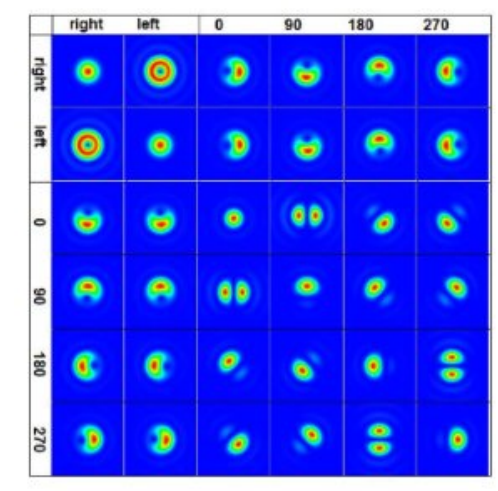

(a)

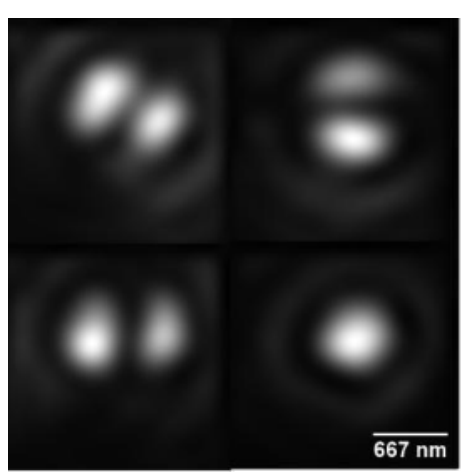

(b)

Figure 1. Table of transfer functions, (a) simulations; (b) Point Spread Functions measured using an EMCCD camera by scanning the illuminations on a $170 \mathrm{~nm}$ fluorescent bead using a microscope with an X40 objective and a 0.95 NA Aperture; the three first three measurements correspond to a rotated "half moons" pattern; the fourth measurement is an Airy disk obtained by the system; the scale bar represent the Airy disk diameter 